(с) М.Н. Пешков ${ }^{1 *}$, Г.П. Пешкова ${ }^{2}$, И.В. Решетов ${ }^{3}$

'Федеральный научно-клинический центр специализированных видов медицинской помощи и медицинских технологий Федерального медико-биологического агентства, Москва

²Рязанский государственный медицинский университет имени академика И.П. Павлова, Рязань

ЗПервый Московский государственный медицинский университет им. И.М. Сеченова (Сеченовский Университет), Москва

Рак предстательной железы является наиболее распространенным видом рака среди мужчин, а андрогенная депривационная терапия (АДТ) - наиболее эффективное лечение этого заболевания. Краеугольным камнем лечения рака предстательной железы является ингибирование выработки тестостерона, которое прерывает вызванный тестостероном рост опухоли предстательной железы. Резкое снижение уровня тестостерона, однако, имеет несколько нежелательных эффектов на метаболический профиль и метаболизм кости, а также может привести к усталости, потере либидо, гинекомастии и анемии, провоцировать вазомоторную гиперемию и в целом влиять на качество жизни. Из-за хорошей (длительной) выживаемости пациентов с раком предстательной железы побочные эффекты, связанные с лечением, имеют большое значение, и поэтому в каждой клинической ситуации преимущества АДТ необходимо сопоставлять с побочными эффектами, связанными с лечением. В настоящей статье основное внимание уделяется описанным метаболическим осложнениям АДТ, включая ожирение, диабет, изменения липидов, метаболический синдром и сердечно-сосудистые заболевания. Также содержатся практические рекомендации по управлению побочными эффектами и осложнениями, основанные на доступных руководствах, выпущенных профессиональными медицинскими сообществами.

КЛЮЧЕВЫЕ СЛОВА: рак предстательной железы; метаболический синдром; сахарный диабет; инсулинорезистентность; индекс массы тела; ожирение; дислипидемия

\title{
METABOLIC CHANGES IN PATIENTS WITH PROSTATE CANCER WITH ANDROGEN DEPRIVATION THERAPY
}

\author{
(c) Maxim N. Peshkov*, Galina P. Peshkova², Igor V. Reshetov ${ }^{3}$
}

${ }^{1}$ Federal Research and Clinical Center of Specialized Medical Care and Medical Technologies FMBA of Russia, Moscow, Russia ${ }^{2}$ Ryazan State Medical University, Ryazan, Russia

3.M. Sechenov First Moscow State Medical Univesity, Moscow, Russia

Prostate cancer is the most common type of cancer among men. Androgen deprivation therapy (ADT) is the most effective treatment for this disease. The cornerstone of prostate cancer treatment is the inhibition of testosterone production, which interrupts testosterone-induced growth of the prostate tumour. A sharp decrease in testosterone, however, has several undesirable effects on the metabolic profile and bone metabolism and can also lead to fatigue, loss of libido, gynecomastia and anaemia, provoke vasomotor hyperaemia and generally affect the quality of life. To increase the good (long-term) survival of patients with prostate cancer, studying the side effects associated with treatment is important, and therefore, in every clinical situation, the benefits of ADT must be compared with the side effects associated with the treatment. This article focuses on the described metabolic complications of ADT, including obesity, diabetes, lipid changes, metabolic syndrome and cardiovascular diseases. It also contains practical recommendations for managing the side effects and complications based on the available guidelines issued by the medical professional community.

KEYWORDS: prostate cancer; metabolic syndrome; diabetes mellitus; insulin resistance; body vass index; obesity; dyslipidemia

Рак предстательной железы является наиболее распространенной опухолью среди мужчин, средний возраст пациентов составляет 68 лет. Эффективное систематическое лечение рака предстательной железы значительно улучшило ожидаемую продолжительность жизни этих пациентов, и, за исключением пациентов с метастатическим заболеванием, 5-летняя канцерспе- цифическая выживаемость составляет сегодня 98,8\% [1]. Андроген-депривационная терапия (АДТ) является фундаментальным элементом лечения нелокализованного рака предстательной железы у пациентов с высоким риском заболевания, которым не предполагается радикальное лечение [2-4]. По оценкам различных авторов, 50\% мужчин с раком предстательной железы проводится 
АДТ на разных этапах лечения [5]. АДТ улучшает общую выживаемость на 26\% за 10 лет наблюдения у пациентов с локализованным и местно-распространенным заболеванием при сочетанном применении с лучевой терапией и использованием внешнего источника излучения [6, 7], хотя у мужчин с метастатическим поражением данная терапия способствует уменьшению боли [8-10]. У пациентов с раком предстательной железы применяют следующие методы АДТ.

\section{Виды андроген-депривационной терапии}

І. Хирургическая АДТ:

- двусторонняя орхэпидэктомия.

II. Фармакологическая АДт:

- терапия агонистами рилизинг-гормона лютеинизирующего гормона (ГнРГ);

- терапия антагонистами ГнРГ;

- эстрогенотерапия;

- максимальная (комбинированная) андрогенная блокада;

- монотерапия антиандрогенами.

Существуют убедительные доказательства увеличения распространенности метаболических изменений (рис. 1) при проведении фармакологической АДТ у пациентов с раком предстательной железы [11].

Метаболический синдром - это совокупность метаболических факторов риска развития сердечно-сосудистых заболеваний и сахарного диабета 2 типа (СД 2). Данные изменения стали более распространенными вместе с ростом ожирения во всем мире [12]. В 1988 г. Reaven описал резистентность к стимулированному инсулином поглощению глюкозы, гиперинсулинемию, увеличение липопротеинов очень низкой плотности, триглицеридемию, снижение липопротеинов высокой плотности (ЛПВП), гипертензию и в совокупности назвал их синдромом X. Reaven предположил, что основной причиной сердечно-сосудистых заболе- ваний является резистентность к инсулину, которая впоследствии приводит к гипертензии, гиперлипидемии и СД $[13,14]$. Термин «метаболический синдром» применяется при наличии трех из следующих пяти факторов: уровень глюкозы в плазме натощак $\geq 6,1$ и <7,0 ммоль/л; уровень триглицеридов в сыворотке крови >1,7 ммоль/л; уровень лПВП в сыворотке крови <1,0 ммоль/л, окружность талии >94 см, артериальное давление $\geq 140 / 90$ мм рт.ст. [15]. Активное медицинское вмешательство, которое предполагает терапию отдельных компонентов этого синдрома, рекомендовано пациентам с наличием данных факторов риска $[3,16]$.

АДТ вызывает метаболические эффекты, которые приводят к изменению структуры тела и липидного профиля, повышают риск развития инсулинорезистентности, диабета и метаболического синдрома [17-19]. В некоторых исследованиях сообщается, что АДТ связана с развитием сердечно-сосудистой заболеваемости и смертности [20-23]. Существует принципиальная схема отличий побочных эффектов и метаболических осложнений АДТ (рис. 2). В данной статье основное внимание уделяется негативным воздействиям АДТ, особенно метаболическим изменениям.

\section{АДТ И МЕТАБОЛИЧЕСКИЙ СИНДРОМ}

В 1941 г. Huggins, Hodges описали преимущества АДТ для пациентов с метастатическим раком простаты. Эта новаторская работа установила четкую связь между уровнем мужских половых гормонов в крови, раком предстательной железы и гормональной терапией как фундаментальной основой лечения [24]. Для пациентов с раком предстательной железы АДТ является методом лечения с доказанным механизмом эффективности [25].

\section{Андроген-депривационная терапия}

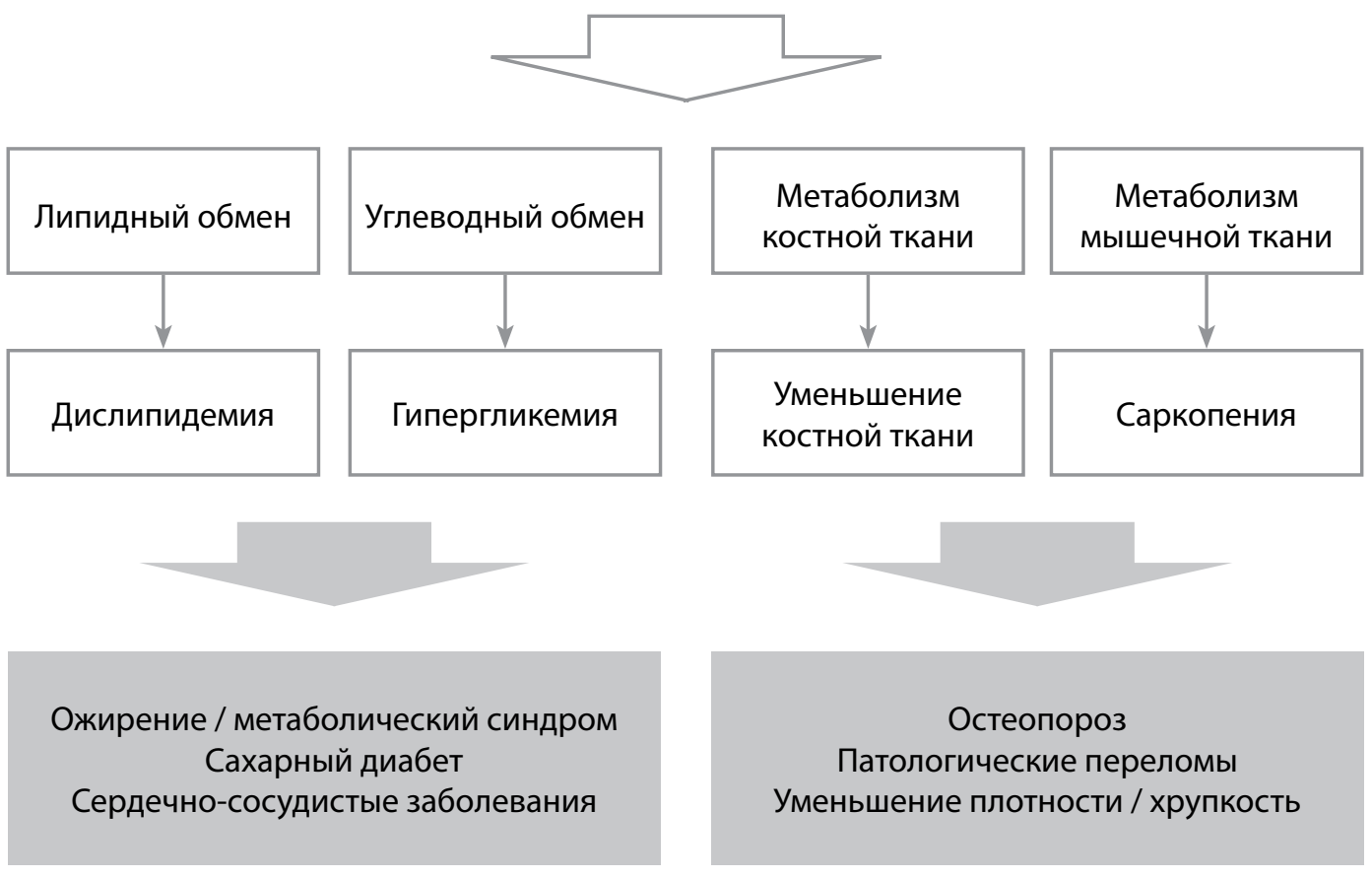

Рис. 1. Метаболические изменения при андроген-депривационной терапии. 


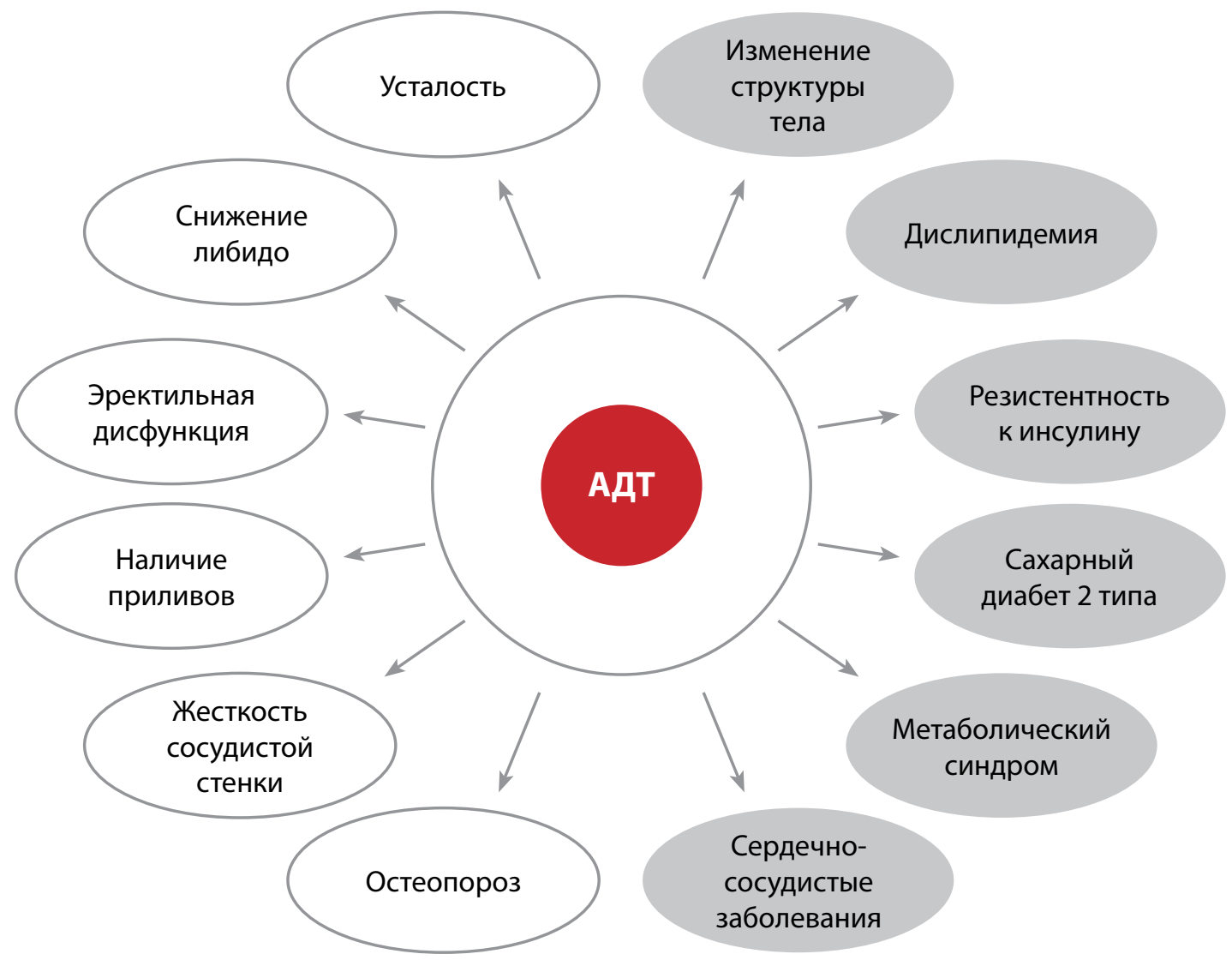

Рис. 2. Побочные эффекты и метаболические осложнения андроген-депривационной терапии.

АДТ вызывает высокую степень изменения метаболических параметров, что увеличивает риск развития метаболического синдрома. Действительно, некоторые исследования показали, что примерно у 36-55\% мужчин, получающих АДТ в течение 12 месяцев и более, развивается метаболический синдром, независимо от возраста, расы и стадии рака предстательной железы [26-28].

Метаанализ, выполненный Alberti и соавт., включающий 9 обсервационных исследований [28] и одно когортное исследование [29], выявил большую распространенность метаболического синдрома - 60-75\% в исследуемой популяции.

Braga-Basaria и соавт. сообщили результаты перекрестного исследования у пациентов с раком предстательной железы, которым проводилась длительная (более 12 месяцев) АДТ; отмечено, что был повышен риск развития резистентности к инсулину и гипергликемии [30]. В данном исследовании проанализированы три группы: пациенты с раком предстательной железы $(\mathrm{n}=18)$, которые находились на АДТ рецидивирующего или метастатического заболевания в течение $\geq 12$ месяцев до включения в исследование; сопоставимые по возрасту мужчины с неметастатическим раком предстательной железы $(\mathrm{n}=17)$ после радикальной простатэктомии и/или лучевой терапии, но без АДТ; пациенты $(\mathrm{n}=18)$ с нормальным уровнем простат-специфического антигена (ПСА) [30]. Мужчины в группе АДТ получали терапию в течение 1-9 лет и имели более высокие уровни глюкозы и инсулина натощак, чем мужчины в двух других группах ( $p=0,002$ и $p=0,002$ соответственно) [30]. Индекс массы тела (ИМТ) также был значительно выше в группе АДТ, чем в двух других группах ( $p=0,005)$. Эти данные позволяют предположить, что у пациентов, которые получают длительную АДТ, могут развиться побочные метаболические эффекты.

АДТ агонистами и антагонистами гонадотропин-рилизинг-гормона (ГнРГ) применяются для проведения терапии неметастатического или рецидивирующего рака после радикального лечения $[25,31]$ вместе с лучевой терапией для лечения первичной опухоли высокой степени прогрессирования. Тестостерон, основной андроген у мужчин, синтезируется в яичках и высвобождается в кровоток, где он связывается с глобулином, связывающим половые гормоны (ГСПГ) [32]. Андроген способствует росту мышечной массы и подавляет отложение жира [32]. Низкий уровень тестостерона и ГСПГ приводит к инсулинорезистентности, увеличивает количество абдоминального жира и частоту центрального ожирения [33]. Исследование пациентов без опухоли подтверждает, что низкий уровень тестостерона может независимо стимулировать инсулинорезистентность и метаболический синдром [34].

Метаболический синдром, возникающий на фоне проведения АДТ, имеет некоторые отличия от классического определения метаболического синдрома. При классическом метаболическом синдроме уровень лПВП снижается примерно на 5\%, в то время как на фоне АДТ отмечается его увеличение $[35,36]$. Метаболический синдром связан с низким уровнем адипонектина [37] 
и повышенным уровнем С-реактивного белка [38], в то время как у пациентов при АДТ уровни адипонектина повышаются, а уровень С-реактивного белка остается неизменным $[39,40]$. Клиническое значение этих различий еще предстоит определить.

Следует отметить, что проведение АДТ приводит к более выраженным изменениям компонентов существующего метаболического синдрома у этих пациентов. Таким образом, скрининг метаболических нарушений до старта АДТ, тщательный мониторинг и корректировка лечения в зависимости от наличия и серьезности метаболических изменений имеют важное значение.

Различные исследования сообщают, что АДТ увеличивает содержание жира и триглицеридов и снижает чувствительность к инсулину $[26,36]$. Однако метаболические изменения, вызванные АДТ, имеют характеристики, отличные от характеристик классического метаболического синдрома, так как АДТ увеличивает ЛПВП и подкожный жир, а не висцеральный абдоминальный жир $[35,36]$. Хотя классический метаболический синдром связан с уменьшением адипонектина [37] и увеличением С-реактивного белка [41], АДТ повышает уровень адипонектина и не меняет уровень С-реактивного белка $[42,36]$. Нами проведено сравнение метаболических эффектов, наблюдаемых при классическом метаболическом синдроме и применении АДТ (табл. 1).

\section{АДТ, ИНСУЛИНОРЕЗИСТЕНТНОСТЬ И САХАРНЫЙ ДИАБЕТ}

Известно, что низкий уровень тестостерона у мужчин связан с резистентностью к инсулину и СД2. На самом деле существует сложная и многопараметрическая корреляция между дефицитом тестостерона и ожирением, это представляет собой фактор риска сердечно-сосудистых заболеваний $[43,44]$. Исследования показали, что уменьшение уровня тестостерона улучшает чувствительность к инсулину и гликемический профиль у мужчин с гипогонадизмом.

Доказательства относительно чувствительности к инсулину у пациентов, получающих АДТ, ограничены, и исследования весьма неоднородны по дизайну (разные типы АДТ, наличие или отсутствие контрольной группы, перманентный или интермиттирующий режим и т. д.). Предполагают, что АДТ снижает чувствитель- ность к инсулину у мужчин без СД в течение 12 недель после начала лечения [45]. Точный механизм, с помощью которого АДТ повышает резистентность к инсулину, не совсем понятен. Считается, что наблюдаемое увеличение жировой массы, особенно в области живота, у этих мужчин способствует снижению чувствительности к инсулину. Более того, было высказано предположение, что некоторые провоспалительные адипокины, такие как TNF-a, IL-6 и резистин, повышены у пациентов с АДТ и могут играть роль в развитии резистентности к инсулину [46].

Резистентность к инсулину появляется на ранней стадии у пациентов, применяющих АДТ. Однако для развития гипергликемии натощак и манифестации с СД требуется более длительный период времени. Рандомизированные исследования по этому вопросу отсутствуют.

Данные из базы данных эпидемиологического надзора и конечных результатов (SEER), включающей 73196 мужчин с локализованным или локо-региональным раком предстательной железы, которым проводилась терапия агонистами ЛгРГ (36\%) или выполнена орхэпидэктомия (7\%), сообщили о более высокой заболеваемости СД (скорректированное отношение рисков 1,42) у этих мужчин [47]. Результаты анализа 20000 пациентов из Канады схожи [48]. Keating и соавт. в обсервационном исследовании с участием 14597 пациентов обнаружили, что лечение агонистами ЛгРГ было связано со статистически значимым увеличением частоты возникновения СД [49].

Ретроспективное когортное исследование 12191 пациентов с диагнозом локализованного рака предстательной железы показало, что АДТ может увеличить риск диабета на 60\% [29]. Обнаружено, что при проведении АДТ ухудшается гликемический контроль, что приводит к увеличению уровня гликированного гемоглобина $\left(\mathrm{HbA}_{1 c}\right)$ у пациентов с ранее существовавшим СД [50, 51].

Поскольку риск развития диабета во время АДТ высок, необходимость скрининга среди мужчин с раком простаты при длительном лечении очевидна. При отсутствии основанных на фактических данных рекомендациий адаптированный к риску скрининг и вмешательство в соответствии с профилактическими принципами могут применяться к группе пациентов на фоне проведения АДТ [52].

Таблица 1. Сравнение метаболического синдрома и метаболических эффектов при андрогенной депривационной терапии.

\begin{tabular}{lcc}
\hline \multicolumn{1}{c}{ Критерий } & Метаболический синдром & Метаболический эффект АДт \\
\hline Телесный жир & Увеличение висцерального жира & Увеличение подкожного жира \\
Обхват талии & Увеличение \\
Соотношение талии и бедер & Увеличение & Увеличение \\
Кровяное давление & Увеличение & Без изменений \\
Триглицерид & Увеличение \\
Липопротеины высокой плотности & Увеличение \\
Липопротеины низкой плотности & Уе определено & Увеличение \\
Адипонектин & Снижение & Увеличение или без изменений \\
С-реактивный белок & Увеличение & Увеличение \\
\hline
\end{tabular}


Скрининг пациентов в самом начале терапии и в течение года для тех, кому проводилась перманентная АДТ с измерением уровня глюкозы в плазме натощак и $\mathrm{HbA}_{1 c}$ в качестве скрининговых тестов, представляется разумным. Пациенты с уровнем $\mathrm{HbA}_{1 c} 6,0-6,5 \%$ или нарушенной глюкозой натощак (глюкоза натощак $\geq 6,1$ и <7,0 ммоль/л) относятся к группе высокого риска развития СД. Данным пациентам рекомендованы похудение и умеренная физическая активность [52]. У пациентов с ранее существовавшим СД может потребоваться интенсификация гипогликемической терапии и более частое наблюдение в зависимости от уровня глюкозы в крови и уровня $\mathrm{HbA}_{1 c^{\circ}}$.

\section{АДТ И ОЖИРЕНИЕ}

Андрогены являются важными детерминантами состава тела у мужчин, способствуя росту мышечной массы и подавляя отложение жира [53]. Эпидемиологические исследования выявили двунаправленную связь между низким уровнем тестостерона и ожирением [54]. Ожирение является единственным наиболее мощным предиктором низкого уровня тестостерона у мужчин, а низкий уровень тестостерона и ГСПГ может предсказать накопление внутрибрюшного жира [55], развитие центрального ожирения (табл. 2) и повышенный риск развития метаболического синдрома [55].

Несколько исследований показали, что у пациентов на фоне проведения АДТ изменения массы тела и состава тела происходят в основном в течение первого года лечения и затем продолжаются в течение 1-2 лет [42]. Сообщалось, что прибавка в весе после года лечения составляет около 1,5-4 кг, и, по-видимому, она выше у более молодых пациентов (<65 лет) и у пациентов без ожирения (ИМТ $<30$ кг/м²) [42]. Изменения в составе тела также происходят рано, с увеличением общего жира до $10 \%$ и уменьшением мышечной массы тела на 2,7\% [35]. Накопление жира в основном подкожное, хотя наблюдается накопление жира как в висцеральном, так и в подкожном слое. Накопление подкожного жира составляет 94\% от общего жира в брюшной полости, полученного в ходе лечения [42]. С другой стороны, уменьшение мышечной массы может привести к снижению мышечной силы и вызвать слабость и усталость [63]. Термин «саркопеническое ожирение» используется в литературе для описания увеличения абдоминального ожирения, сопровождаемого снижением мышечной массы у пациентов с АДТ [64], связанного с уменьшением общей выживаемости [65].

Основные механизмы увеличения жира в брюшной полости при АДТ недостаточно изучены. Известно, что висцеральные адипоциты имеют рецепторы андрогенов; было высказано предположение, что тестостерон вовлечен в мобилизацию жирных кислот [66]. Изменения в распределении жира при АДТ связаны с потерей андроген-опосредованного ингибирования дифференцировки стволовых клеток в адипоциты [67]. Высказано предположение, что накопление висцерального жира у этих мужчин может быть связано со снижением липолиза в результате низкого уровня тестостерона [68].

Таблица 2. Изменения в составе тела при андроген-депривационной терапии
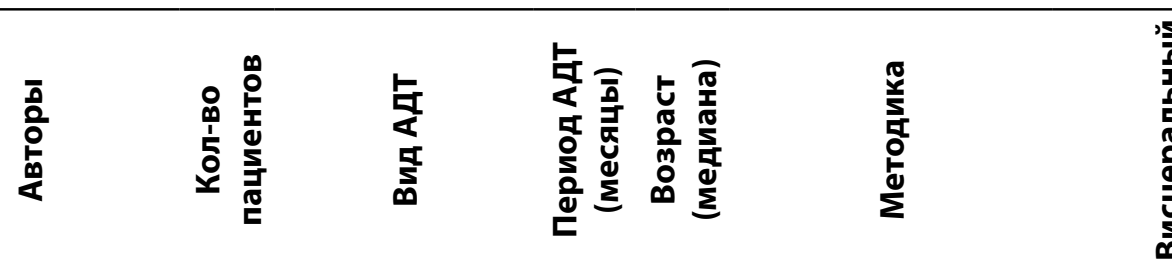

Lee $H$. et al.

[56]

65 Агонисты ГнРГ $12 \quad 66$

Рентгеновская

абсорбциометрия

$+6,6 \%$

(массы жира)
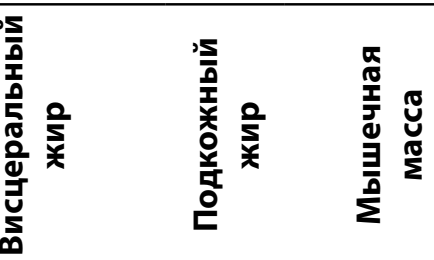

Smith M.R. et al.

[57]

25 Агонисты ГнРГ С

$1268+0,6$ кг $(0,4 \%)$

$+4,3 \%$

(массы жира)

$-2,0 \%$

NA (мышечной мaсcы)

$-1,4 \%$

$+23,0$ (мышечной массы)

\begin{tabular}{|c|c|c|c|c|c|c|c|c|}
\hline $\begin{array}{l}\text { Hamilton E.L. } \\
\text { [58] }\end{array}$ & 26 & NA & 12 & 71 & KT (L 4-5) & $+22,0$ & $+13,0$ & NA \\
\hline $\begin{array}{l}\text { Torimoto K. } \\
\text { [59] }\end{array}$ & 39 & $\begin{array}{c}\text { Агонисты } \\
\text { ГнРГ с/без } \\
\text { Бикалутамидом }\end{array}$ & 12 & 74 & $\begin{array}{c}\text { Биоэлектрический } \\
\text { импедансный } \\
\text { анализ }\end{array}$ & $\begin{array}{c}+2,08 \text { кг } \\
\text { (массы жира) } \\
+17,24 \text { см }^{2} \\
\text { (висцерального } \\
\text { жира) }\end{array}$ & $\mathrm{NA}$ & $+0,57 \mathrm{kг}$ \\
\hline $\begin{array}{l}\text { Smith J.C. et al. } \\
{[60]}\end{array}$ & 22 & $\begin{array}{c}\text { Агонисты ГнРГ } \\
(21) \\
\text { Орхэктомия (1) }\end{array}$ & 3 & 67 & $\begin{array}{c}\text { Биоэлектрический } \\
\text { импедансный } \\
\text { анализ }\end{array}$ & $\begin{array}{c}+8,4 \text { кг } \\
\text { (массы жира) }\end{array}$ & NA & $\begin{array}{c}-2,7 \% \text { кг } \\
\text { (мышечной } \\
\text { массы) }\end{array}$ \\
\hline $\begin{array}{l}\text { Cheng A.S. et al. } \\
{[61]}\end{array}$ & 34 & NA & 12 & 68 & $\begin{array}{c}\text { Рентгеновская } \\
\text { абсорбциометрия }\end{array}$ & $\begin{array}{c}\text { +3,5 кг } \\
\text { (массы жира) } \\
\text { Не изменено } \\
\text { (висцерального } \\
\text { жира) }\end{array}$ & $\mathrm{NA}$ & $-1,49$ кг \\
\hline
\end{tabular}




\begin{tabular}{|c|c|c|c|c|c|c|c|c|c|c|c|c|}
\hline $\begin{array}{l}\bar{a} \\
\frac{0}{0} \\
\frac{1}{\alpha}\end{array}$ & 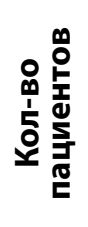 & 皆 & 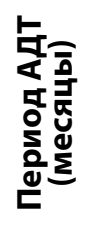 & 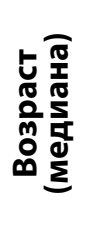 & Фّँ & 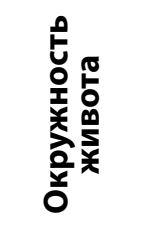 & 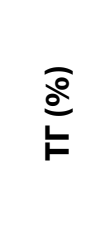 & 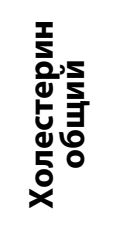 & $\begin{array}{l}\text { 둔 } \\
\text { 듣 }\end{array}$ & 듬 & 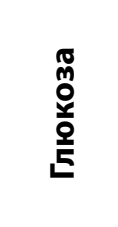 & $\begin{array}{l}\frac{\partial}{9} \\
\frac{0}{1} \\
\frac{0}{1}\end{array}$ \\
\hline $\begin{array}{l}\text { Smith M.R. et al. } \\
\text { [57] }\end{array}$ & 25 & $\begin{array}{c}\text { Агонисты ГнРГ } \\
\text { с Бикалутамидом }\end{array}$ & 12 & 68 & $\begin{array}{l}+0,6 \mathrm{Kr} \\
(0,4 \%)\end{array}$ & NA & $+23,0$ & $+9,4$ & $+8,7$ & $+9,9$ & $+2,0$ & $+2,9$ \\
\hline $\begin{array}{l}\text { Hamilton E.L. } \\
\text { [58] }\end{array}$ & 26 & NA & 12 & 71 & $\begin{array}{l}+1,8 \mathrm{Kr} \\
(2,3 \%)\end{array}$ & $+1,9 \%$ & $+46,6$ & $+11,2$ & $+9,0$ & $+2,3$ & $+0,3$ & $+3,0$ \\
\hline $\begin{array}{l}\text { Torimoto K. } \\
\text { [59] }\end{array}$ & 39 & $\begin{array}{c}\text { Агонисты } \\
\text { ГнРГ с / без } \\
\text { Бикалутамида }\end{array}$ & 12 & 74 & $+1,37 \mathrm{kr}$ & $+2,0 \mathrm{~cm}$ & $+14,1$ & $+4,1$ & $+14,8$ & $-3,2$ & NA & NA \\
\hline $\begin{array}{l}\text { Salvador C. et al. } \\
\text { [62] }\end{array}$ & 33 & $\begin{array}{c}\text { Агонисты } \\
\text { ГнРГ с / без } \\
\text { Бикалутамида }\end{array}$ & 12 & 73 & NA & NA & $+3,8$ & $+7,6 \%$ & $+0,9$ & $-14,7$ & NA & NA \\
\hline $\begin{array}{l}\text { Smith J.C. et al. } \\
\text { [60] }\end{array}$ & 22 & $\begin{array}{c}\text { Агонисты ГнРГ } \\
(21) \\
\text { Орхэктомия (1) }\end{array}$ & 3 & 67 & $\begin{array}{c}\text { Без } \\
\text { изменений }\end{array}$ & NA & $+13,3$ & $3,2 \%$ & $-2,3 \%$ & $+8,3 \%$ & $+3,5 \%$ & NA \\
\hline
\end{tabular}

В настоящее время отсутствуют фактические данные о стратегии профилактики или эффективной терапии изменений в составе тела, побочных эффектов и осложнений, связанных с проведением АДТ. Однако существуют убедительные доказательства в отношении пациентов с прогрессирующим заболеванием при АДТ, которые свидетельствуют о том, что физические упражнения эффективны для улучшения качества жизни, уменьшения усталости и увеличения физической работоспособности, характерных для рака [69]. В исследовании Culos-Reed пациенты, которые увеличили свою физическую активность, имели положительные изменения при измерении окружности талии и бедра, снижение артериального давления [70]. Имеются данные, свидетельствующие о том, что 6-месячная комбинированная программа аэробных упражнений и упражнений с отягощениями оказывает значительное благоприятное воздействие на окисление жиров в покое, глюкозу и состав тела, несмотря на проводимую АДТ [71].

\section{АДТ И ДИСЛИПИДЕМИЯ}

В нескольких работах выявлены изменения липидного профиля мужчин при АДТ, но результаты не являются последовательными и зависят от продолжительности терапии и метода АДТ [72]. В некоторых исследованиях количество изучаемых параметров было небольшим. Хотя результаты не являются универсальными, АДТ, по-видимому, повышает уровни общего холестерина, липопротеинов низкой плотности (ЛПНП), ЛПВП и триглицеридов $[26,68,72,73]$. Исследования показали значительное увеличение уровней триглицеридов (примерно на 26\%), общего холестерина (примерно на 10\%) и ЛПВП (примерно на 8-10\%), но без изменений в уровнях ЛПнП после 3, 6 и 12 месяцев АДТ [68, 73, 74].

Гиперинсулинемия может быть причиной некоторых изменений в липидном профиле мужчин, полу- чающих АДТ, но не может объяснить изменения холестерина и ЛПВП (табл. 3). Следовательно, могут быть вовлечены некоторые другие факторы, например, повышение уровня адипонектина у этой категории пациентов. Адипонектины считаются атеротромботическими факторами риска у лиц с ожирением, так как связаны с гемостатическими маркерами и толщиной стенки общей сонной артерии [75]. Однако влияние этих изменений на сердечно-сосудистый риск неизвестно, и необходимы дальнейшие исследования для установления этой взаимосвязи [68, 73]. У пациентов на фоне АДТ изменения диеты и образа жизни рекомендуются в качестве вмешательств первой линии, для достижения концентраций ЛПНП на целевом уровне если присутствует гиперлипидемия. Если эти меры не дают результатов, рекомендуется медикаментозная терапия статинами [76]. Обнаружено, что селективные модуляторы рецепторов эстрогена (Selective estrogen receptor modulator, SERMs), которые применяются для воздействия на минеральную плотность костной ткани (Bone Mineral Density, BMD) и предотвращения переломов у пациентов при АДТ, также влияют на уровень липидов в сыворотке. В рандомизированном плацебо-контролируемом исследовании торемифен назначался для профилактики переломов у пациентов на фоне АДТ, и при плановом контрольном исследовании через 12 месяцев выявлено снижение уровня холестерина, ЛПнП, триглицеридов и повышение уровня лПВП [77].

\section{ЗАКЛЮЧЕНИЕ}

В настоящее время хорошо известно, что АДТ улучшает выживаемость у мужчин с раком предстательной железы, однако это сопровождается появлением метаболических изменений, которые, в свою очередь, приводят к ожирению, инсулинорезистентности и СД, дислипидемии и сердечно-сосудистым заболеваниям. 
Пациенты должны быть хорошо информированы об этих рисках, и потенциальные преимущества терапии должны быть тщательно рассмотрены до начала АДТ. Оптимальное управление этими метаболическими изменениями еще не определено. Увеличение физической активности, потеря веса и изменение образа жизни очень важны для лечения этих метаболических нарушений и должны быть рекомендованы всем пациентам, подвергающимся АДТ. Необходимы дальнейшие исследования, нацеленные на выработку идеальной стратегии отбора пациентов для АДТ и оптимального способа лечения побочных эффектов АДТ.

\section{ДОПОЛНИТЕЛЬНАЯ ИНФОРМАЦИЯ}

Финансирование работы. Обзорно-аналитическая работа по подготовке рукописи проведена на личные средства автора.

Конфликт интересов. Обзор является частью диссертационного исследования М.Н. Пешкова. Остальные авторы декларируют отсутствие явных и потенциальных конфликтов интересов, связанных с публикацией настоящей статьи.

Участие авторов: Пешков М.Н. - сбор, анализ данных литературы, написание статьи; Пешкова Г.П., Решетов И.В. - редактирование статьи. Все авторы прочли и одобрили финальную версию рукописи перед публикацией.

\section{СПИСОК ЛИТЕРАТУРЫ | REFERENCES}

1. Ryan CJ, Small EJ. Early versus delayed androgen deprivation for prostate cancer: new fuel for an old debate. J Clin Oncol. 2005;23(32):8225-8231. doi: https://doi.org/10.1200/JCO.2005.03.5311

2. Каприн А.Д., Троянов А.В., Иванов С.А., Карякин О.Б. Комплексное гормонохимиолучевое лечение больных неметастатическим раком предстательной железы группы высокого и очень высокого риска прогрессирования // Онкоурология. 2017. - T. 13. - №1. - C. 101-111. [Kaprin AD, Troyanov AV, Ivanov SA, Karyakin OB. Combined chemohormonalradiation treatment of highand very-high-risk non-metastatic prostate cancer. Onkouro. 2017;13(1):101-111. (In Russ.)] doi: https://doi.org/10.17650/1726-9776-2017-13-1-101-111

3. Алексеев Б.Я. Новые возможности комбинированной антиандрогенной терапии больных метастатическим гормоночувствительным раком предстательной железы // Онкоурология. - 2019. - Т. 15. — №3. - С. 89-101. [Alekseev BY. New possibilities of combined antiandrogen therapy in patients with metastatic hormone-sensitive prostate cancer. Onkouro. 2019;15(3):89-101. (In Russ.)] doi: https://doi.org/10.17650/1726-9776-2019-15-3-89-101

4. Шестакова М.В., Чазова И.Е., Шестакова Е.А. Российское многоцентровое скрининговое исследование по выявлению недиагностированного сахарного диабета 2 типа у пациентов с сердечно-сосудистой патологией // Сахарный диабет. - 2016. T. 19. - №1. - C. 24-29. [Shestakova MV, Chazova IE, Shestakova EA. Russian multicentre type 2 diabetes screening program in patients with cardiovascular disease. Diabetes mellitus. 2016;19(1):24-29 (In Russ.)] doi: https://doi.org/10.14341/DM7765

5. Shahinian VB, Kuo Yf, Freeman JL, et al. Increasing use of gonadotropin releasing hormone agonists for the treatment of localized prostate carcinoma. Cancer. 2005;103(8):1615-1624. doi: https://doi.org/10.1002/cncr.20955.

6. Heidenreich A, Bastian PJ, Bellmunt J, et al. EAU guidelines on prostate cancer. part 1: screening, diagnosis, and local treatment with curative intent-update 2013. Eur Urol. 2014;65(1):124-137. doi: https://doi.org/10.1016/j.eururo.2013.09.046

7. Bolla $M$, Collette $L$, Blank $L$, et al. Long term results with immediate androgen suppression and external irradiation in patients with locally advanced prostate cancer (an EORTC study): a phase III randomized trial. Lancet. 2002;360(9327):103-106. doi: https://doi.org/10.1016/s0140-6736(02)09408-4

8. Messing EM, Manola J, Yao J, et al. Immediate versus deferred androgen deprivation treatment in patients with nodepositive prostate cancer after radical prostatectomy and pelvic lymphadenectomy. Lancet Oncol. 2006;7(6):472-479. doi: https://doi.org/10.1016/S1470-2045(06)70700-8

9. Huggins $C$, Hodges CV. Studies on prostate cancer: II. The effects of castration on advanced carcinoma of the prostate gland. Arch Surg. 1941;43(2):209-233. doi: https://doi.org/10.1001/archsurg.1941.01210140043004

10. Heidenreich A, Bastian PJ, Bellmunt J, et al. EAU guidelines on prostate cancer. Part II: Treatment of advanced, relapsing, and castration-resistant prostate cancer. Eur Urol. 2014;65(2):467-479. doi: https://doi.org/10.1016/j.eururo.2013.11.002

11. Saylor PJ, Smith MR. Metabolic complications of androgen deprivation therapy for prostate cancer. J Urol. 2009;181(5):1998-2006. doi: https://doi.org/10.1016/j.juro.2009.01.047
12. Eckel RH, Grundy SM, Zimmet PZ. The metabolic syndrome. Lancet. 2005;365(9468):1415-1428. doi: https://doi.org/10.1016/S0140-6736(05)66378-7

13. Reaven GM. Banting lecture 1988. Role of insulin resistance in human disease. Diabetes. 1988;37(12):1595-1607. doi: https://doi.org/10.2337/diab.37.12.1595

14. Kahn R, Buse J, Ferrannini E, Stern M. The metabolic syndrome: time for a critical appraisal. Joint statement from the American diabetes association and the european association for the study of diabetes. Diabetologia. 2005;48(9):2289-2304 doi: https://doi.org/10.2337/diacare.28.9.2289

15. Executive summary of the third report of the national cholesterol education program (ncep) expert panel on detection, evaluation, and treatment of high blood cholesterol in adults (adult treatment panel III). JAMA. 2001;285(19):2486-2497. doi: https://doi.org/10.1001/jama.285.19.2486

16. Gallagher EJ, Leroith D, Karnieli E. The metabolic syndrome - from insulin resistance to obesity and diabetes. Med Clin North Am. 2011;95(5):855-873. doi: https://doi.org/10.1016/j.mcna.2011.06.001

17. Saylor PJ, Smith MR. Metabolic complications of androgen deprivation therapy for prostate cancer. J Urol. 2013;189(1 Suppl):S34-42. doi: https://doi.org/10.1016/j.juro.2012.11.017

18. Nobes JP, Langley SE, Laing RW. Metabolic syndrome and prostate cancer: a review. Clin Oncol (R Coll Radiol). 2009;21(3):183-191. doi: https://doi.org/10.1016/j.clon.2008.11.013

19. Leahy Y. Risk of metabolic syndrome, cardiovascular disease, and diabetes in androgen deprivation therapy. Clin J Oncol Nurs. 2008;12(5):771-776. doi: https://doi.org/10.1188/08.CJON.771-776

20. Saigal CS, Gore JL, Krupski TL, et al. Androgen deprivation therapy increases cardiovascular morbidity in men with prostate cancer. Cancer. 2007;110(7):1493-1500. doi: https://doi.org/10.1002/cncr.22933

21. Efstathiou JA, Bae K, Shipley WU, et al. Cardiovascular mortality after androgen deprivation therapy for locally advanced prostate cancer: RTOG 85-31. J Clin Oncol. 2009;27(1):92-99. doi: https://doi.org/10.1200/JCO.2007.12.3752

22. Van Poppel H, Tombal B. Cardiovascular risk during hormonal treatment in patients with prostate cancer. Cancer Manag Res. 2011;3:49-55. doi: https://doi.org/10.2147/CMR.S16893

23. Roayaei M, Ghasemi S. Effect of androgen deprivation therapy on cardiovascular risk factors in prostate cancer. J Res Med Sci. 2013;18(7):580-582

24. Huggins C, Hodges CV. Studies on prostatic cancer: I. The effect of castration, of estrogen and of androgen injection on serum phosphatases in metastatic carcinoma of the prostate. 1941. J Urol. 2002;168(1):9-12. doi: https://doi.org/10.1016/s0022-5347(05)64820-3

25. Roach M. 3rd. Current trends for the use of androgen deprivation therapy in conjunction with radiotherapy for patients with unfavorable intermediate-risk, high-risk, localized, and locally advanced prostate cancer. Cancer. 2014;120(11):1620-1629. doi: https://doi.org/10.1002/cncr.28594

26. Braga-Basaria M, Dobs AS, Muller DC, et al. Metabolic syndrome in men with prostate cancer undergoing long-term androgendeprivation therapy. J Clin Oncol. 2006;24(24):3979-3983. doi: https://doi.org/10.1200/JCO.2006.05.9741 
27. Munoz GJ, Samper OP, Rios KY, et al. Cross-section observational study about prevalence of metabolic syndrome and osteoporosis in prostate cancer treated with androgen-deprivation therapy and their impact on quality of life (SIMBOSPROST): Gicor Group. Intl J Rad Onc Bio Phys. 2013;87(2):356. doi: https://doi.org/10.1016/j.jijrobp.2013.06.934

28. Alberti KG, Zimmet P, Shaw J. The metabolic syndrome: a new worldwide definition. Lancet. 2005;366(9491):1059-1062. doi: https://doi.org/10.1016/S0140-6736(05)67402-8

29. Tsai HT, Keating NL, van Den Eeden SK, et al. Risk of diabetes among patients receiving primary androgen deprivation therapy for clinically localized prostate cancer. J Urol. 2015;193(6):1956-1962. doi: https://doi.org/10.1016/j.juro.2014.12.027

30. Basaria S, Muller DC, Carducci MA, et al. Hyperglycemia and insulin resistance in men with prostate carcinoma who receive androgen-deprivation therapy. Cancer. 2006;106(3):581-588. doi: https://doi.org/10.1002/cncr.21642

31. Bastian PJ, Boorjian SA, Bossi A, et al. High-risk prostate cancer: from definition to contemporary management. Eur Urol. 2012;61(6):1096-1106. doi: https://doi.org/10.1016/j.eururo.2012.02.031

32. Yu IC, Lin HY, Sparks JD, et al. Androgen receptor roles in insulin resistance and obesity in males: the linkage of androgen-deprivation therapy to metabolic syndrome. Diabetes. 2014;63(10):3180-3188. doi: https://doi.org/10.2337/db13-1505

33. Vermeulen A, Goemaere S, Kaufman JM. Testosterone, body composition and aging. J Endocrinol Invest. 1999;22(5 Suppl):110-116. doi: https://doi.org/10.3109/13685539909003178

34. Laaksonen DE, Niskanen L, Punnonen K, et al. Testosterone and sex hormone-binding globulin predict the metabolic syndrome and diabetes in middle-aged men. Diabetes Care. 2004;27(5):1036-1041. doi: https://doi.org/10.2337/diacare.27.5.1036

35. Smith MR, Finkelstein JS, McGovern FJ, et al. Change in body composition during androgen deprivation therapy for prostate cancer. J Clin Endocrinol Metab. 2002;87(2):599-603. doi: https://doi.org/10.1210/jcem.87.2.8299

36. Smith MR, Lee $H, M c G o v e r n ~ F$, et al. Metabolic changes during gonadotropin-releasing hormone agonist therapy for prostate cancer: Differences from the classic metabolic syndrome. Cancer. 2008;112(10):2188-2194. doi: https://doi.org/10.1002/cncr.23440

37. Trujillo ME, Scherer PE. Adiponectin journey from an adipocyte secretory protein to biomarker of the metabolic syndrome. J Intern Med. 2005;257(2):167-175. doi: https://doi.org/10.1111/j.1365-2796.2004.01426.x

38. Dockery F, Bulpitt CJ, Agarwal S, et al. Testosterone suppression in men with prostate cancer leads to an increase in arterial stiffness and hyperinsulinemia. Clin Sci (London). 2003;104(2):195-201. doi: https://doi.org/10.1042/cs20020209

39. Hamilton EJ, Gianatti E, Strauss BJ, et al. Increase in visceral and subcutaneous abdominal fat in men with prostate cancer treated with androgen deprivation therapy. Clin Endocrinol. 2011;74(3):377-383. doi: https://doi.org/10.1111/j.1365-2265.2010.03942.x

40. Scailteux LM, Naudet F, Alimi Q. Mortality, cardiovascular risk, and androgen deprivation therapy for prostate cancer: A systematic review with direct and network meta-analyses of randomized controlled trials and observational studies. Medicine (Baltimore). 2016;95(24):e3873. doi: https://doi.org/10.1097/MD.0000000000003873

41. Haffner SM. The metabolic syndrome: inflammation, diabetes mellitus, and cardiovascular disease. Am J Cardiol. 2006;97(2A):3A-11A. doi: https://doi.org/10.1016/j.amjcard.2005.11.010

42. Smith MR, Lee H, Fallon MA, Nathan DM. Adipocytokines, obesity, and insulin resistance during combined androgen blockade for prostate cancer. Urology. 2008;71(2):318-322. doi: https://doi.org/10.1016/j.urology.2007.08.035

43. Traish AM, Zitzmann M. The complex and multifactorial relationship between testosterone deficiency (TD), obesity and vascular disease. Rev Endocr Metab Disord. 2015;16(3):249-268. doi: https://doi.org/10.1007/s11154-015-9323-2

44. Ullah MI, Washington T, Kazi M, et al. Testosterone deficiency as a risk factor for cardiovascular disease. Horm Metab Res. 2011;43(3):153-164. doi: https://doi.org/10.1055/s-0030-1270521

45. Keating NL, O'Malley AJ, Smith MR. Diabetes and cardiovascular disease during androgen deprivation therapy for prostate cancer. J Clin Oncol. 2006;24(27):4448-4456. doi: https://doi.org/10.1200/JCO.2006.06.2497
46. Cutolo M, Sulli A, Capellino S, et al. Sex hormones influence on the immune system: basic and clinical aspects in autoimmunity. Lupus. 2004;13(9):635-638. doi: https://doi.org/10.1191/0961203304lu1094oa

47. Smith RM. Androgen deprivation therapy and risk for diabetes and cardiovascular disease in prostate cancer survivors. Curr Urol Rep. 2008;9(3):197-202. doi: https://doi.org/10.1007/s11934-008-0035-y

48. Alibhai SM, Duong-Hua M, Sutradhar R, et al. Impact of androgen deprivation therapy on cardiovascular disease and diabetes. J Clin Oncol. 2009;27(21):3452-3458. doi: https://doi.org/10.1200/JCO.2008.20.0923

49. Keating NL, O'Malley AJ, Freedland SJ, Smith MR. Diabetes and cardiovascular disease during androgen deprivation therapy: observational study of veterans with prostate cancer. J Nat/ Cancer Inst. 2010;102(1):39-46. doi: https://doi.org/10.1093/jnci/djp404

50. Keating NL, Liu PH, O'Malley AJ, et al. Androgendeprivation therapy and diabetes control among diabetic men with prostate cancer. Eur Urol. 2014;65:816-824. doi: https://doi.org/10.1016/j.eururo.2013.02.023

51. Morote J, Gomez-Caamano A, Alvarez-Ossorio JL, et al. The metabolic syndrome and its components in patients with prostate cancer on androgen deprivation therapy. J Urol. 2015;193(6):1963-1969. doi: https://doi.org/10.1016/j.juro.2014.12.086

52. American Diabetes Association. Standards of medical care in diabetes-2008. Diabetes Care. 2008;31 Suppl 1:S12-54. doi: https://doi.org/10.2337/dc08-S012

53. Finkelstein JS, Lee H, Burnett-Bowie SA, et al. Gonadal steroids and body composition, strength, and sexual function in men. N Engl J Med. 2013;12;369(11):1011-1022. doi:https://doi.org/10.1056/nejmoa1206168

54. Allan CA, McLachlan RI. Androgens and obesity. Curr Opin Endocrinol Diabetes Obes. 2010;17(3):224-232. doi: https://doi.org/10.1097/MED.0b013e3283398ee2

55. Brand JS, van der Tweel I, Grobbee DE, et al. Testosterone, sex hormone-binding globulin and the metabolic syndrome: a systematic review and meta-analysis of observational studies. Int J Epidemiol. 2011;40(1):189-207. doi: https://doi.org/10.1093/ije/dyq158

56. Lee H, McGovern K, Finkelstein JS, Smith MR. Changes in bone mineral density and body composition during initial and longterm gonadotropinreleasing hormone agonist treatment for prostate carcinoma. Cancer. 2005;104(8):1633-1637. doi: https://doi.org/10.1002/cncr.21381

57. Smith MR, Lee $H$, Nathan DM. Insulin sensitivity during combined androgen blockade for prostate cancer. J Clin Endocrinol Metab. 2006;91(4):1305-1308. doi: https://doi.org/10.1210/jc.2005-2507

58. Hamilton EJ, Gianatti E, Strauss BJ et al. Increase in visceral and subcutaneous abdominal fat in men with prostate cancer treated with androgen deprivation therapy. Clin Endocrinol (Oxf). 2011;74(3):377-383. doi: https://doi.org/10.1111/j.1365-2265.2010.03942.x

59. Torimoto K, Samma S, Kagebayashi Y, et al. The effects of androgen deprivation therapy on lipid metabolism and body composition in Japanese patients with prostate cancer. Jpn J Clin Oncol. 2011:41(4):577-581. doi: https://doi.org/10.1093/jjco/hyr005

60. Smith JC, Bennett S, Evans LM, et al. The effects of induced hypogonadism on arterial stiffness, body composition, and metabolic parameters in males with prostate cancer. J Clin Endocrinol Metab. 2001;86(9):4261-4267. doi: https://doi.org/10.1210/jcem.86.9.7851

61. Cheung AS, Hoermann R, Dupuis P, et al. Relationships between insulin resistance and frailty with body composition and testosterone in men undergoing androgen deprivation therapy for prostate cancer. Eur J Endocrinol. 2016;175(3):229-237. doi: https://doi.org/10.1530/EJE-16-0200

62. Salvador C, Planas J, Agreda F, et al. Analysis of the lipid profile and atherogenic risk during androgen deprivation therapy in prostate cancer patients. Urol Int. 2013;90(1):41-44. doi: https://doi.org/10.1159/000342814

63. Stone $\mathrm{P}$, Hardy J, Huddart $\mathrm{R}$, et al. Fatigue in patients with prostate cancer receiving hormone therapy. Eur J Cancer. 2000;36(9):1134-1141. doi: https://doi.org/10.1016/s0959-8049(00)00084-8

64. Zamboni M, Mazzali G, Fantin F, et al. Sarcopenic obesity: a new category of obesity in the elderly. Nutr Metab Cardiovasc Dis. 2008;18(5):388-395. doi: https://doi.org/10.1016/j.numecd.2007.10.002 
65. Tian S, XuY. Association of sarcopenic obesity with the risk of all-cause mortality: A meta-analysis of prospective cohort studies. Geriatr Gerontol Int. 2016;16(2):155-166. doi: https://doi.org/10.1111/ggi.12579

66. Reis C, Liberman S, Pompeo AC, et al. Body composition alterations, energy expenditure and fat oxidation in elderly males suffering from prostate cancer, pre- and post-orchiectomy. Clinics (Sao Paulo). 2009:64(8):781-784. doi: https://doi.org/10.1590/S1807-59322009000800012

67. Chazenbalk G, Singh P, Irge D, et al. Androgens inhibit adipogenesis during human adipose stem cell commitment to preadipocyte formation. Steroids. 2013;78(9):920-926. doi: https://doi.org/10.1016/j.steroids.2013.05.001

68. Smith JC, Bennett S, Evans LM, et al. The effects of induced hypogonadism on arterial stiffness, body composition, and metabolic parameters in males with prostate cancer. J Clin Endocrinol Metab. 2001;86(9):4261-4267. doi: https://doi.org/10.1210/jcem.86.9.7851

69. Bourke L, Smith D, Steed L, et al. Exercise for men with prostate cancer: A systematic review and meta-analysis. Eur Urol. 2016;69(4):693-703. doi: https://doi.org/10.1016/j.eururo.2015.10.047

70. Culos-Reed SN, Robinson JW, Lau H, et al. Physical activity for men receiving androgen deprivation therapy for prostate cancer: benefits from a 16-week intervention. Support Care Cancer. 2010;18(5):591-599. doi: https://doi.org/10.1007/s00520-009-0694-3

71. Wall BA, Galvão DA, Fatehee N, et al. Exercise improves $\mathrm{VO}_{2 \text { MAX }}$ and body composition in ADT-treated prostate cancer patients. Med Sci Sports Exerc. 2017:49(8):1503-1510. doi: https://doi.org/10.1249/MSS.0000000000001277
72. Shahani S, Braga-Basaria M, Basaria S. Androgen deprivation therapy in prostate cancer and metabolic risk for atherosclerosis. J Clin Endocrinol Metab. 2008;93(6):2042-2049. doi: https://doi.org/10.1210/jc.2007-2595

73. Lewington S, Clarke R, Qizilbash N, et al. Age-specific relevance of usual blood pressure to vascular mortality: a meta-analysis of individual data for one million adults in 61 prospective studies. Lancet. 2002;360(9349):1903-1913. doi: https://doi.org/10.1016/S0140-6736(02)11911-8

74. Rezaei MM, Rezaei MM, Ghoreifi A, Kerigh BF. Metabolic syndrome in patients with prostate cancer undergoing intermittent androgendeprivation therapy. Can Urol Assoc J. 2016;10(9-10):E300-E305. doi: https://doi.org/10.5489/cuaj.3655

75. Csongrádi É, Káplár M, Nagy B Jr, et al. Adipokines as atherothrombotic risk factors in obese subjects: Associations with haemostatic markers and common carotid wall thickness. Nutr Metab Cardiovasc Dis. 2017;27(6):571-580. doi: https://doi.org/10.1016/j.numecd.2017.02.007

76. Wilt TJ, Bloomfield HE, MacDonald R, et al. Effectiveness of statin therapy in adults with coronary heart disease. Arch Intern Med. 2004;164(13):1427-1436. doi: https://doi.org/10.1001/archinte.164.13.1427

77. Smith MR, Malkowicz SB, Chu F, et al. Toremifene increases bone mineral density in men receiving androgen deprivation therapy for prostate cancer: interim analysis of a multicenter phase 3 clinical study. J Urol. 2008;179(1):152-155. doi: https://doi.org/10.1016/j.juro.2007.08.137

\section{ИНФОРМАЦИЯ ОБ АВТОРАХ [AUTHORS INFO]}

*Пешков Максим Николаевич, к.м.н. [Maxim N. Peshkov, MD, PhD]; адрес: Россия, 125371, Москва, Волоколамское ш., д. 91 [address: 91, Volokolamskoye Highway street, 125371 Moscow, Russia]; ORCID: https://orcid.org/0000-0002-1497-8646; eLibrary SPIN: 1691-1478; e-mail: drpeshkov@gmail.com

Пешкова Галина Петровна, к.M.н. [Galina P. Peshkova, MD, PhD]; ORCID: https://orcid.org/0000-0002-3336-4025; eLibrary SPIN: 6849-5407; e-mail: gppeshkova@gmail.com

Решетов Игорь Владимирович, Д.м.H. [Igor V. Reshetov, MD, PhD]; ORCID: https://orcid.org/0000-0002-5739-3170; eLibrary SPIN: 3845-6604; e-mail: reshetoviv@mail.ru

\section{ЦИТИРОВАТЬ:}

Пешков М.Н., Пешкова Г.П., Решетов И.В. Метаболические изменения у пациентов с раком предстательной железы при андроген депривационной терапии (обзор) // Сахарный диабет. — 2020. — Т. 23. — №2. — С. $192-200$. doi: https://doi.org/10.14341/DM10343

\section{TO CITE THIS ARTICLE:}

Peshkov M.N., Peshkova G.P., Reshetov IV. Metabolic changes in patients with prostate cancer with androgen deprivation therapy (review). Diabetes Mellitus. 2020;23(2):192-200. doi: https://doi.org/10.14341/DM10343 